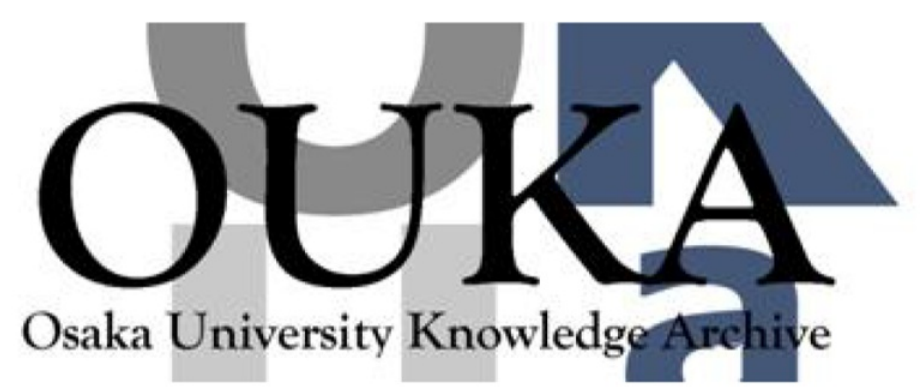

\begin{tabular}{|c|l|}
\hline Title & $\begin{array}{l}\text { Optical properties and laser action in one- } \\
\text { dimensional periodic dielectrics containing } \\
\text { Liquid crystal as defect layer }\end{array}$ \\
\hline Author(s) & Ozaki, R.; Matsui, T.; Miyoshi, H. et al. \\
\hline Citation & $\begin{array}{l}\text { Proceedings of the IEEE International } \\
\text { Conference on Properties and Applications of } \\
\text { Dielectric Materials. 2 p.528-p.531 }\end{array}$ \\
\hline Issue Date & 2003-06 \\
\hline oaire:version & VoR \\
\hline URL & $\begin{array}{l}\text { https://hdl. handle. net/11094/14073 } \\
\text { c2003 IEEE. Personal use of this material is } \\
\text { permitted. However, permission to } \\
\text { reprint/republish this material for advertising } \\
\text { or promotional purposes or for creating new } \\
\text { collective works for resale or redistribution } \\
\text { to servers or lists, or to reuse any } \\
\text { copyrighted component of this work in other } \\
\text { works must be obtained from the IEEE. }\end{array}$ \\
\hline rights \\
\hline Note & \begin{tabular}{l} 
\\
\hline
\end{tabular} \\
\hline
\end{tabular}

Osaka University Knowledge Archive : OUKA

https://ir. Library. osaka-u. ac. jp/

Osaka University 


\title{
Optical Properties and Laser Action in One-dimensional Periodic Dielectrics Containing Liquid Crystal as Defect Layer
}

\author{
R. Ozaki", T. Matsui, H. Miyoshi, M. Ozaki and K. Yoshino \\ Department of Electric Engineering Graduate School of Engineering, Osaka University, \\ Yamada-Oka 2-1, Suita, Osaka 565-0871, Japan \\ *E-mail: rozaki@ele.eng.osaka-u.ac.jp
}

\begin{abstract}
The wavelength of the laser action based on a defect mode in a one-dimensional periodic structure has been electrically controlled using a dye-doped nematic liquid crystal as a defect layer. The lasing wavelength is widely tuned upon the applying electric field, which originates from the refractive index change in the defect layer caused by the field-induced realignment of the liquid crystal molecules.
\end{abstract}

\section{INTRODUCTION}

Photonic crystal (PC) having a three-dimensional (3-D) ordered structure with a periodicity of optical wavelength has attracted considerable attention from both fundamental and practical points of view, because in such materials a photonic band gap (PBG) exists in which the existence of a certain energy range of photons is forbidden [1]-[2]. Novel physical concepts such as PBG have been theoretically predicted and various applications of PCs have been proposed. Especially, the study on a defect mode in PBG is one of the most attractive subjects, since photons are localized and low threshold laser based on the defect mode of the PCs should be expected. However a complete 3-D PC with a periodicity equivalent to visible wavelength remains a technical challenge [3]. Not only 3-D PCs but also one-dimensional (1-D) PCs are an attractive subject. Although, the 1-D PC does not have a complete PBG, there are plenty of applications using extraordinary dispersion of the photon and localized photonic state in a defect layer. So far, intensive studies on 1-D PC applications have been reported: the air-bridge microcavities [4], the photonic bandedge laser [5]-[7], the nonlinear optical diode [8] and the enhancement of optical nonlinearity [9][10].

On the other hand, liquid crystals (LCs) have a large optical anisotropy and are sensitive to an external stress such as an electric field. Based on such optical anisotropy and field sensitivity, a tunable photonic crystal has been proposed in opal or inverse opal infiltrated with LC [11]-[16]. Although opal and inverse opal are simple and inexpensive approach to realize a 3-D PC using selforganization of colloidal particles [17]-[18], the introduction of defects into the 3-D periodic structure is a problem that must be resolved.
Recently we have introduced a LC layer in a dielectric multilayer structure as a defect in 1-D PC [19], in which the wavelength of defect modes were controlled upon applying electric field in a basis of the change in optical length of the defect layer caused by the field-induced molecular reorientation of a LC.

In this paper, we report the wavelength tunable laser based on an electrically controllable defect mode in a 1-D dielectric periodic structure containing a dye-doped LC as a defect layer. The lasing in a 1-D PC with LC defect layer can be tuned in a wide range upon the applying electric field.

\section{EXPERIMENTAL}

The 1-D PC with a LC defect is shown in Fig. 1. A dielectric multilayer consisting of an alternating stack of $\mathrm{SiO}_{2}$ and $\mathrm{TiO}_{2}$ layers deposited on an In-Sn oxide (ITO) coated glass substrate is used as the 1-D PC. The center wavelength of the photonic band is adjusted to be $600 \mathrm{~nm}$ by setting the optical thickness of both $\mathrm{SiO}_{2}$ and $\mathrm{TiO}_{2}$ to be one-quarter of $600 \mathrm{~nm}$. The refractive indices of $\mathrm{SiO}_{2}$ and $\mathrm{TiO}_{2}$ are 1.46 and 2.35, respectively, and the thickness of $\mathrm{SiO}_{2}$ and $\mathrm{TiO}_{2}$ layers are $103 \mathrm{~nm}$ and $64 \mathrm{~nm}$, respectively. The number of $\mathrm{SiO}_{2}-\mathrm{TiO}_{2}$ pairs on each substrate is 5 . The top surface of the dielectric multilayer is coated with a polyimide (Japan Synthetic Rubber AL1254) and unidirectionally rubbed along the $z$-axis in Fig. 1 .

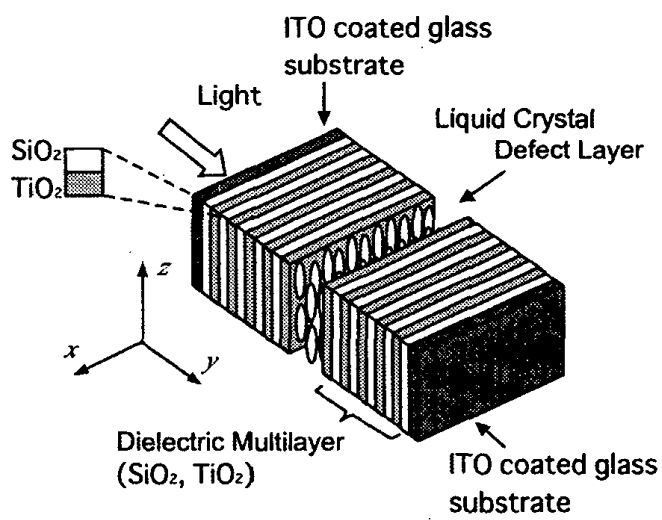

Fig.1 One-dimensional photonic crystal with liquid crystal defect layer. 
In order to introduce the defect layer, a dyedoped nematic liquid crystal (Merck, E47) was sandwiched between substrates with a dielectric multilayer using $2 \mu \mathrm{m}$ spacers. The refractive index anisotropy $\Delta \mathrm{n}$ of $\mathrm{E} 47$ is 0.209 at room temperature. In the absence of an electric field, the long molecular axis of the LC is aligned parallel to the substrates ( $z$-axis). As a laser dye doped in the LC, [2-[2-4-(dimethylamino) phenyl] ethenyl]-6-methyl4H-pyran-4-ylidene] propanedinitrile, DCM (Exciton) is used. The concentration of the dye is 0.5 wt.-\%.

A rectangular wave voltage of $1 \mathrm{kHz}$ was applied between ITO layers to change the molecular alignment of the LC in the defect layer. In order to investigate the characteristics of defect mode, the transmission spectrum of the linearly polarized light propagating along the $y$-axis was measured from the opposite side of the cell using a charge-coupled device (CCD) multi-channel spectrometer (Hamamatsu photonics, PMA-11) having a spectral resolution of $3 \mathrm{~nm}$.

A second harmonic light of a Q-switched Nd:YAG laser (Spectra Physics, Quanta-Ray INDI) is used for an excitation, whose wavelength, pulse width and pulse repetition frequency are $532 \mathrm{~nm}, 8 \mathrm{~ns}$ and $10 \mathrm{~Hz}$, respectively. The illumination area on the sample is about $0.2 \mathrm{~mm}^{2}$. The excitation laser beam irradiated the sample perpendicularly to the cell plate. The emission spectra from the 1-D PC with dye-doped nematic LC are measured from the opposite side of the cell using the CCD multichannel spectrometer. In order to control the emission wavelength, the orientation of the LC molecules in the defect layer was changed upon applying a rectangular wave voltage of $1 \mathrm{kHz}$.

\section{RESULTS AND DISCUSSIONS}

Figure 2 (a) shows the transmission spectrum of a 10-pairs dielectric multilayer without a defect. Figure 2 (b) shows the transmission spectra for the incident light polarized along the $z$-axis which corresponds to the rubbing direction and the initial orientation direction of the LC molecules in the defect layer, as a function of the applied voltage. The solid and broken lines correspond to spectra at 0 $\mathrm{V}$ and $1.2 \mathrm{~V}$, respectively. Regardless of the application of voltage, PBGs are observed in the spectral range from $520 \mathrm{~nm}$ to $780 \mathrm{~nm}$ and these bandwidths do not depend on the voltage. The slight decrease in the transmittance at shorter wavelengths is attributed to the absorption of DCM. The peaks of the defect modes shift to shorter wavelengths upon the applying voltage. This peak shift originates from the decrease in the optical length of the defect layer caused by the field-induced reorientation of the LC molecules [19]. Therefore, we confirmed that the wavelength of the defect mode in the 1-D PC with
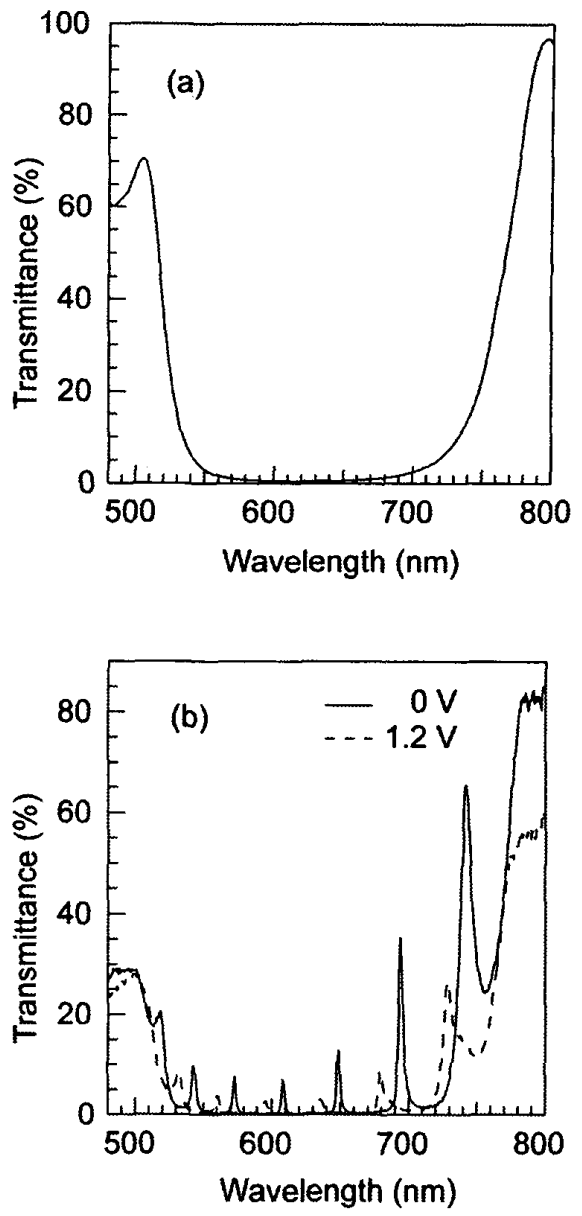

Fig. 2 (a) Transmission spectrum of a 10-pair dielectric multilayer without a defect.

(b) Transmission spectra of a dielectric multilayer with DCM-doped LC defect as a function of the applied voltage.

the dye-doped LC layer can be controlled upon the applying voltage.

We have performed a theoretical calculation of the light propagation in the periodic multilayer structure containing a defect layer using a method of characteristic matrices. This method is a numerical analysis of Maxwell equation which can quantitatively calculate the light propagation in the medium with the refractive index varying along one direction. The light propagating along the $y$ - axis with frequency $\omega$ is given by the next equation

$$
\frac{d \psi(y)}{d y}=\frac{i \omega}{c} \mathrm{D}(y) \psi(y),
$$

where $\mathrm{D}(y)$ is a derivative propagation matrix and $\Psi(y)=\left(E_{x}, H_{z}, E_{z}, H_{x}\right)$. In this calculation, ITO is neglected. 
Figure 3 (b) shows the calculated transmission spectrum of a dielectric multilayer with a LC defect whose refractive index and width are $n_{d}=1.7$ and 2.3 $\mu \mathrm{m}$, respectively. For $n_{d}=1.7$ corresponding to $n_{e}$ of the LC used in this study, 7 peaks of defect modes appeared. Comparing with the experimental result shown in Fig 3 (a), the calculated spectra for $n_{d}=$ 1.7 is quite similar to the experimental spectra at 0 $V$. It is found that optical distances of the experimental defect layer are $3.91 \mu \mathrm{m}$ at $0 \mathrm{~V}$. Theoretically calculated defect modes wavelength is good agreement with experimental one. Furthermore the peak level of defect modes qualitatively agrees between the experimental and the calculated values.

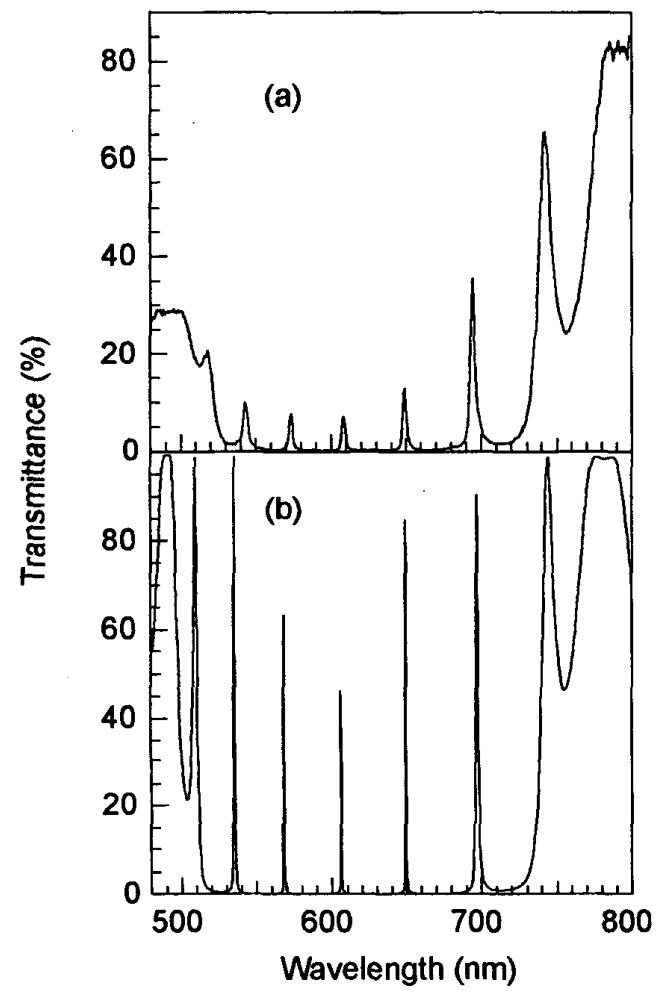

Fig. 3 (a) Transmission spectrum of a dielectric multilayer with a DCM-doped LC defect at 0V. (b) Calculated transmission spectrum of a dielectric multilayer with a LC defect layer whose refractive index and thickness are 1.7 and $2.3 \mu \mathrm{m}$, respectively.

Figure 4 (a) shows photoluminescence spectrum of the DCM-doped LC without a 1-D PC. Figure 4(b) shows the emission spectra of the 1-D PC with the DCM-doped LC defect layer as a function of the applied voltage. A sharp emission peak appears. Lasing wavelength was tuned with the applied voltage.

Figure 5 shows the pump energy dependence of the

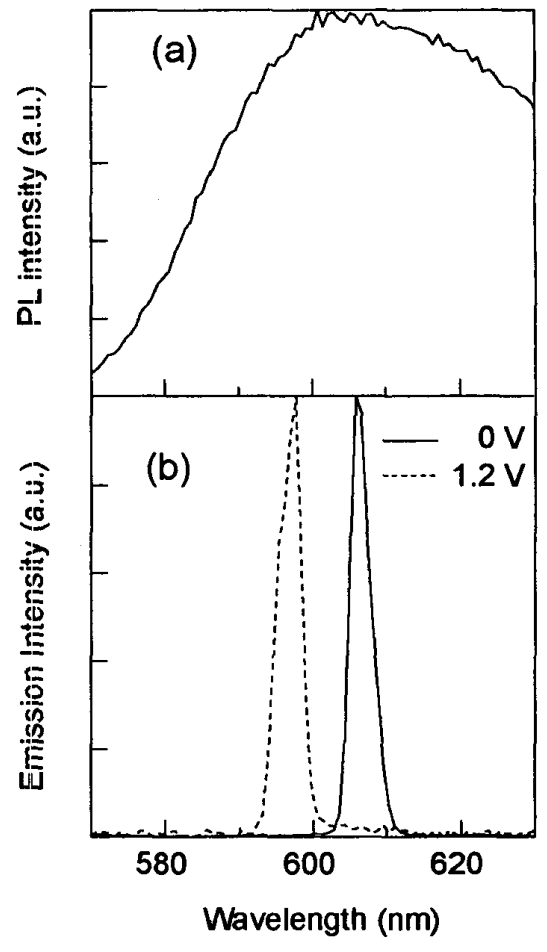

Fig. 4 (a) Photoluminescence spectrum of the DCM-doped LC without a 1-D PC.

(b) Emission spectra of the DCM-doped LC defect layer included in a 1-D PC as a function of the applied voltage.

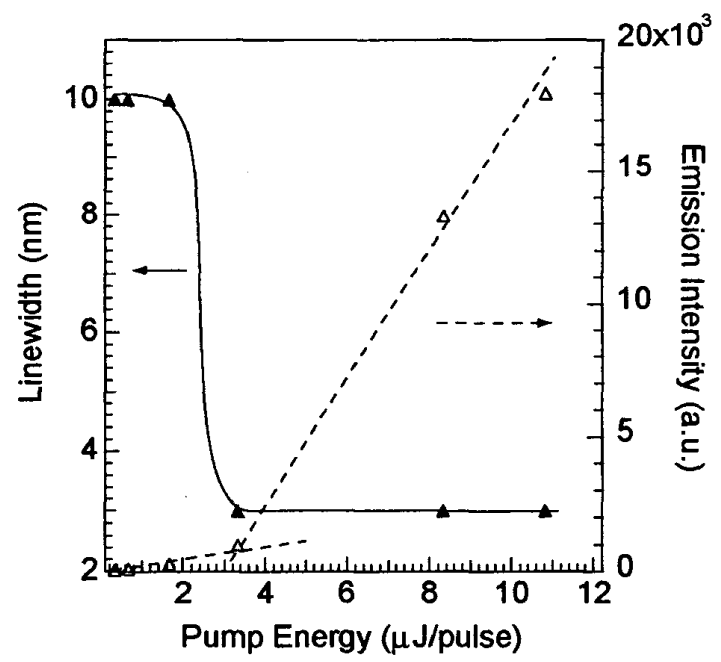

Fig. 5 Pump energy dependence of the peak intensity and linewidth of the emission spectrum of the 1-D PC with LC defect.

peak intensity. This indicates that there exists a lasing threshold. Above the threshold at a pump pulse energy of about $3 \mu \mathrm{J}$, the emission intensity 
drastically increases. The full width at half maximum (FWHM) of the lasing peak is about $3 \mathrm{~nm}$, which is limited by the spectral resolution of the CCD spectrometer used in this experiment. The FWHM of this emission peak is narrower than that of the transmission peak of the defect mode $(10 \mathrm{~nm})$ shown in Fig. 2 (b). This indicates that the emission peak in the band is not the spontaneous emission of the DCM passing through the narrow band window of a defect mode. It should be noted that lasing peak shifts toward shorter wavelengths with increasing voltage as the same manner as the defect modes shift shown in Fig. 2 (b). The wavelength shift of the lasing peak is about $10 \mathrm{~nm}$ even upon the applying low voltage.

Figure 6 shows the voltage dependence of the lasing peak wavelength. As is evident from Fig. 6, a threshold voltage exists for the peak shift and the lasing wavelength shifts toward shorter wavelengths above the threshold of $0.9 \mathrm{~V}$. This is associated with Fredericks transition of the LC in the defect layer. In Fig. 6, the discontinuous change of the lasing wavelength is observed around $1.3 \mathrm{~V}$. This means that the defect mode at shorter wavelengths go out of the wavelength range of the high emission efficiency of a DCM above $1.3 \mathrm{~V}$. On the other hand, the defect mode peak at the longer wavelengths appears in the lasing range above $1.7 \mathrm{~V}$.

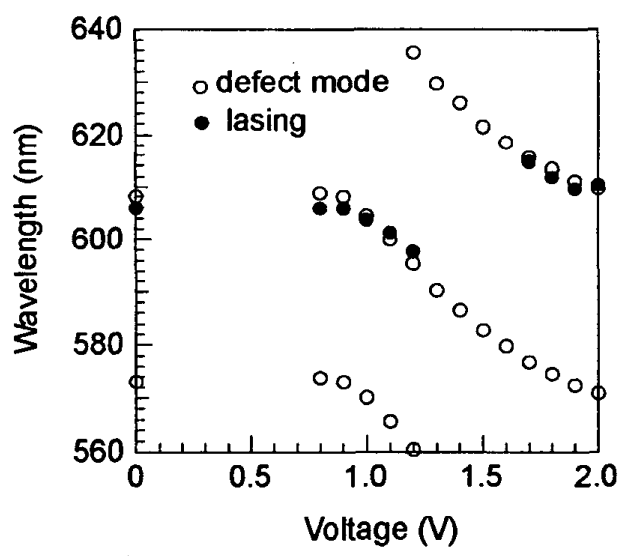

Fig. 6 Voltage dependence of the defect mode and the lasing wavelength.

\section{CONCLUSIONS}

The electrical tuning of the defect mode lasing in a 1-D PBG was demonstrated using a dye-doped nematic liquid crystal as a defect layer. Laser emission was observed upon the irradiation of pump laser beam above the threshold energy. Lasing wavelength was widely tuned with a low voltage.

\section{ACKNOWLEDGEMENTS}

This work is supported by a Grant-in-Aid for Scientific Research (14350165) from the Japan Ministry of Education, Culture, Sports, Science and Technology.

\section{REFERENCES}

[1] E. Yablonovitch, Phys. Rev. Lett., Vol.58, pp.2059-2062, 1987.

[2] S. John, Phys. Rev. Lett., Vol.58, pp.2486-2489, 1987.

[3] S. Noda, K. Tomoda, N. Yamamoto and A. Chutinan, Science, vol.289, pp. 604-606, 2000.

[4] J. S. Foresi, P. R. Villeneuve, J. Ferrera, E. R. Thoen, G. Steinmeyer, S. Fan, J. D. Joannopoulos, L. C. Kimerling, H. I. Smith and E. P. Ippen, Nature, vol.390, pp.143-145, 1997.

[5] J. P. Dowling, M. Scalora, M. J. Bloemer and C. M. Bowden, J. Appl. Phys., vol.75, pp.1896-1899, 1994.

[6] V. 1. Kopp, B. Fan, H. K. M. Vithana, and A. Z. Genack, Opt. Lett., vol.23, pp.1707- 1709, 1998.

[7] M. Ozaki, M. Kasano, D. Ganzke, W. Haase and K.Yoshino, Adv.Mater., vol.14, pp.306-309, 2002.

[8] M. D. Tocci, M. J. Bloemer, M. Scalora, J. P. Dowling and C. M. Bowden, Appl. Phys. Lett., vol.66, pp.2324-2326, 1995.

[9] T.Hattori, N. Tsurumachi and H. Nakatsuka, J. Opt. Soc. Am., vol.14, pp.348-355, 1997.

[10] Y. Dumeige, P. Vidakovic, S. Sauvage, I. Sgnes and J. A. Levenson, Appl. Phys. Lett., vol.78, pp.3021-3023, 2001.

[11] K. Yoshino, S. Satoh, Y. Shimoda, Y. Kawagishi, K. Nakayama and M. Ozaki, Jpn. J. Appl. Phys., Vol.38, pp.L961-L963, 1999.

[12] K. Yoshino, Y. Shimoda, Y. Kawagishi, K. Nakayama and M. Ozaki, Appl. Phys. Lett., Vol.75, pp.932-934, 1999.

[13] K. Busch and S. John, Phys. Rev., Lett. Vol.83, pp.967 $-970,1999$.

[14] Y. Shimoda, M. Ozaki and K. Yoshino, Appl. Phys. Lett., vol.79, pp.3627-3629, 2001.

[15] D. Kang, J. E. Maclennan, N. A. Clark, A. A. Zakhidov and R. H. Baughman, Phys. Rev. Lett. 86, 4052 (2001).

[16] M. Ozaki, Y. Shimoda, M. Kasano and K.Yoshino, Adv. Mater., vol.14, pp.514-518, 2002.

[17] K. Yoshino, K. Tada, M. Ozaki, A. A. Zakhidov and R. H. Baughman, Jpn. J. App. Phys., vol.36, pp.L7 14-L717, 1997.

[18] Y. A. Vlasov, K. Luterova, I. Pelant, B. Honerlage and V. N. Astratov, Appl. Phys. Lett., vol71, pp.1616-1618, 1997.

[19] R. Ozaki, T. Matsui, M. Ozaki and K. Yoshino, Jpn. J. App. Phys., vol.41, pp.L1482-1484, 2002. 